\title{
Functional outcomes after laparoscopic versus robotic-assisted rectal resection: a systematic review and meta-analysis
}

\author{
K. F. Kowalewski ${ }^{1,2} \cdot$ L. Seifert $^{1}$ - S. Ali ${ }^{1} \cdot$ M. W. Schmidt ${ }^{1} \cdot$ S. Seide ${ }^{3} \cdot$ C. Haney ${ }^{1} \cdot$ C. Tapking ${ }^{1} \cdot$ A. Shamiyeh ${ }^{4} \cdot$ Y. Kulu $^{1}$. \\ T. Hackert ${ }^{1}$ • B. P. Müller-Stich ${ }^{1}$ · F. Nickel ${ }^{1}$ (1)
}

Received: 11 September 2019 / Accepted: 24 December 2019 / Published online: 5 February 2020

(c) The Author(s) 2020

\begin{abstract}
Surgical resection is crucial for curative treatment of rectal cancer. Through multidisciplinary treatment, including radiochemotherapy and total mesorectal excision, survival has improved substantially. Consequently, more patients have to deal with side effects of treatment. The most recently introduced surgical technique is robotic-assisted surgery (RAS) which seems equally effective in terms of oncological control compared to laparoscopy. However, RAS enables further advantages which maximize the precision of surgery, thus providing better functional outcomes such as sexual function or contience without compromising oncological results. This review was done according to the PRISMA and AMSTAR-II guidelines and registered with PROSPERO (CRD42018104519). The search was planned with PICO criteria and conducted on Medline, Web of Science and CENTRAL. All screening steps were performed by two independent reviewers. Inclusion criteria were original, comparative studies for laparoscopy vs. RAS for rectal cancer and reporting of functional outcomes. Quality was assessed with the Newcastle-Ottawa scale. The search retrieved 9703 hits, of which 51 studies with 24,319 patients were included. There was a lower rate of urinary retention (non-RCTs: Odds ratio (OR) [95\% Confidence Interval (CI)] 0.65 [0.46, 0.92]; RCTs: OR[CI] 1.29[0.08, 21.47]), ileus (non-RCTs: OR[CI] 0.86[0.75, 0.98]; RCTs: OR[CI] 0.80[0.33, 1.93]), less urinary symptoms (non-RCTs mean difference (MD) $[C I]-0.60[-1.17,-0.03]$; RCTs: $-1.37[-4.18,1.44]$ ), and higher quality of life for RAS (only non-RCTs: $\mathrm{MD}[\mathrm{CI}]: 2.99$ [2.02, 3.95]). No significant differences were found for sexual function (non-RCTs: standardized MD[CI]: 0.46[- 0.13, 1.04]; RCTs: SMD[CI]: 0.09[- 0.14, 0.31]). The current meta-analysis suggests potential benefits for RAS over laparoscopy in terms of functional outcomes after rectal cancer resection. The current evidence is limited due to non-randomized controlled trials and reporting of functional outcomes as secondary endpoints.
\end{abstract}

Keywords Minimally invasive surgery $\cdot$ Laparoscopy $\cdot$ Robotic-assisted surgery $\cdot$ Rectal cancer $\cdot$ Functional outcomes · Meta-analysis · Evidence-based medicine

K. F. Kowalewski and L. Seifert have contributed equally to this work.

Electronic supplementary material The online version of this article (https://doi.org/10.1007/s00464-019-07361-1) contains supplementary material, which is available to authorized users.

F. Nickel

felix.nickel@med.uni-heidelberg.de

1 Department of General, Visceral, and Transplantation Surgery, University of Heidelberg, Im Neuenheimer Feld 110, 69120 Heidelberg, Germany

2 Department of Urology, University Medical Center Mannheim, Heidelberg University, Theodor-Kutzer Ufer 1-3, 68167 Mannheim, Germany

\section{Background}

Colorectal Cancer is currently the third most common cancer in the world and the rectum is affected in approximately one-third of cases [1]. The mainstay of curative treatment is surgical resection with lymph node dissection

3 Institute for Medical Biometry and Informatics, Heidelberg University, Im Neuenheimer, Feld 130.3, 69120 Heidelberg, Germany

4 Klinik für Allgemein-Und Viszeralchirurgie, Kepler Universitätsklinikum GmbH, Med Campus III., Krankenhausstraße 9, 4021 Linz, Austria 
and total or partial mesorectal excision (TME/PME) [2, $3]$. Depending on tumor stage localization surgery can be accompanied by (neo)adjuvant (radio)chemotherapy [4] .

Surgery can be performed by either traditional open surgery or minimally invasive approaches. International multicenter studies such as the COLOR II trial [5] or COREAN $[6,7]$ have proven the equivalence of laparoscopy and open surgery for oncological outcomes (e.g., 5-year disease-free survival), while ALaCaRT [8] and ACOSOG [9] showed equivocal results. Additionally, a current meta-analysis confirmed these findings [10]. Furthermore, robotic-assisted surgery (RAS) has been recently implemented in the field of general surgery for performing complex procedures in rectal [11], pancreatic [12] or esophageal surgery [13]. Advantages include 3D vision, a steady camera and the endowrist function with seven degrees of freedom. As for rectal cancer in particular, a recent meta-analysis found no differences between conventional laparoscopy and RAS in terms of oncological outcomes [14]. Additionally, another meta-analysis compared open, RAS, laparoscopy, and transanal TME and found that laparoscopy and RAS enhance postoperative recovery, while open surgery and transanal TME may improve oncological resection [15].

In combination with screening methods, the available treatment options have led to a lower mortality for rectal cancer in the western world over the last decades [16, 17], with an improvement of 5-year survival from $48.1 \%$ in the 1970 s to now $67.7 \%$ [18]. Due to these higher survival rates, there is now a considerably higher number of patients with long-term survival that have to deal with potential side effects of the treatment which can have a profound impact on patient's quality of life (QoL). These influencing factors include but are not limited to bowel and urinary continence, sexual function as well as the immediate postoperative course.

Even in the established fields of RAS such as urology, there is no clear evidence indicating better oncologic outcomes for RAS when compared to laparoscopic surgery (or open surgery) [19]. However, there is evidence that shows an improvement in functional outcomes for RAS [7, 8]. This might be true for rectal cancer as well. It is frequently hypothesized that the technological advancements of the robotic system might facilitate fine dissection in anatomical planes and could thus improve the intactness of nerves and other structures relevant for functional outcomes. Current studies focus mainly on oncologic outcomes or perioperative parameters [14] or raise considerable concerns for methodologic quality, e.g., without critical appraisal of the quality of evidence or bias analysis [20,21].

Therefore, the aim of this review is to compare functional outcomes for laparoscopic and RAS for rectal cancer.

\section{Materials and methods}

This systematic review was conducted in line with the Preferred Reporting Items for Systematic Reviews and MetaAnalyses (PRISMA) statement [22] and the AMSTAR 2 criteria [23]. In addition, it was prospectively registered with PROSPERO (CRD42018104519).

\subsection{Search strategy and information sources}

A comprehensive database search including Medline (via PubMed), Web of Science and CENTRAL was performed as suggested by Goossen et al. [24] until November 2018. However, more recent trials which were identified by manual search during the working process were also included if eligible. No restrictions in terms of language, year or study design were applied. Assistance of a librarian from Heidelberg University was sought to optimize the search strategy based on PICO criteria [25]:

$\mathbf{P}$ (patients)-Male or female patients over the age of 18 with disease of the rectum which requires elective rectal resection.

I (intervention)—Robotic-assisted rectal resection.

C (comparator)—Conventional laparoscopic rectal resection.

$\mathbf{O}$ (outcome) - At least one functional outcome.

$\mathbf{S}$ (Study design) - Comparative studies with all types of study designs.

RCTs and cohort studies were eligible since RCTs are often criticized to not necessarily reflect reality as there are always patient and surgeon specific factors which influence the choice of treatment. However, they were also analyzed separately in order to evaluate whether different study types lead to different outcomes. Furthermore, different types of resection such as AR, ISR, or APR were included. Depending on the study, high and/or low AR were reported together.

\subsection{Screening process and data extraction}

Two reviewers independently screened titles/abstracts and consecutively full-text of eligible studies. Disagreement was solved by consensus or a third reviewer. Data from included studies were extracted into a dedicated data sheet and pre-tested to prove its suitability. In addition, references and grey literature (e.g., abstracts) were searched. In case of similar studies from the same research groups or missing data, authors were contacted to evaluate whether studies report on the same patient clientele and to gather additional data. For example, in order to gain more information from the ROLARR trial [11] for urinary and sexual dysfunction, an official data sharing agreement were signed. In case of 
multiple publications of one study, the main manuscript was included, but all of the manuscripts were read and included for data extraction in order to gather all available information.

\subsection{Data items}

Data were sought for (1) general information (e.g., year of publication, country), (2) study participant characteristics (e.g., age, disease, BMI) and inclusion/exclusion criteria, (3) type of intervention [laparoscopic vs. robotic-assisted, as well as type of resection (AR, APR, ISR)] and (4) functional outcomes [ileus, urinary retention, international index erectile function (IIEF), international prostate symptom score (IPSS) which includes typical lower urinary tract (frequency, urgency, straining, intermittency, weak stream, nocturia, incomplete emptying), Quality of life scores (QoL)]. Furthermore, analysis was stratified by study design (RCT vs. non-RCT). In case of different follow-up intervals or time periods, the value of the last available follow-up time point was used for analysis.

\subsection{Inclusion criteria}

- Original studies.

- Reporting functional outcomes.

- Comparative studies (robotic vs laparoscopic rectal resection).

\subsection{Exclusion criteria}

- Indication other than rectal cancer.

- No stratified analysis by study arm.

- Studies before 2001 (after the first reported robotic procedure for colorectal cancer) [26].

- Redundant patient population.

- Juvenile studies.

- Cadavers.

\subsection{Risk of bias in individual studies}

Risk of bias in the individual studies was assessed independently by two reviewers with the Newcastle-Ottawa scale (NOS) [27]. Disagreements regarding the risk of bias assessment were resolved with a third party. Publication bias was assessed with the help of funnel plots.

\subsection{Certainty in evidence}

Certainty in evidence was assessed using the GRADE approach [28]. This was done independently by two reviewers using the GRADE Pro Software (McMaster University and Evidence Prime Inc, Ontario, Canada). In case of disagreement a third reviewer was consulted.

\subsection{Statistical analysis}

Statistical analysis was performed by a biometrician (S.S.) at the Department of Medical Biometry and Informatics at Heidelberg University who was otherwise not involved in the study.

Endpoints were either binary or (quasi) continuous. In case of binary data, the odds ratio were used as effect measure and trials were pooled using the Mantel-Haenzel method to account for the sparse number of events [29, 30]. In case of 0 event either trial arm, 0.5 was added as continuity correction to both arms of the trial. The continuous endpoints were mainly estimated by the mean in each trial arm at pre-surgery and different follow-up times. We used the mean difference as effect measure for continuous endpoints, except for the IIEF total score, where trials reported different measures and the standardized mean difference estimated by Hedges' $g$ was used. If studies reported medians and interquartile ranges, the mean was estimated by the median the standard deviation by the width of the interquartile range divided by 1.35 [31]. For all meta-analyses, randomeffects models with the DerSimonian-Laird estimator for between-trial heterogeneity were estimated to account for unexplained variation. Continuous endpoints were pooled using the inverse variance method.

The individual trial results were additionally combined within type of trial as pre-specified subgroups to account for potential bias in the observational trials. Heterogeneity was explored and reported by the $I^{2}$-statistic. Funnel plot asymmetry was assessed graphically for all endpoints, however, no test on funnel plot asymmetry was performed as the presence of subgroups on trial type may already have introduced asymmetry.

The analyses were carried out in the software $\mathrm{R}$ (version 3.5.1) [32] and its extension meta (version 4.9.2) [33].

\subsection{Institutional review board}

Approval by an internal review board was not applicable since only data which were already published was used for this study.

\section{Results}

\subsection{Study selection}

A total of 9703 studies were screened for eligibility (see PRISMA flowchart Fig. 1). After abstract and full-text screening 51 studies were included for qualitative and 
Fig. 1 PRISMA flow diagram Identification

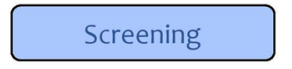

Eligibility
48 studies for quantitative analyses reporting on 24,319 patients. General information about studies such as year, author, country of origin as well as baseline characteristics are listed in Table 1.

\subsection{Outcomes}

\subsubsection{Ileus}

There was a total of 34 studies (31 non-RCTs, 3 RCTs, 21,452 patients) that reported on ileus. There was a significant reduction of the odds of developing an ileus postoperatively in favor of RAS compared to the laparoscopic
Additional record identified through other sources $(n=1)$

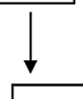

Records after exclusion of

1) Duplicates $(n=163)$

2) Records before

$2001^{*}(n=2648)$

(Total excluded $n=2811$ )

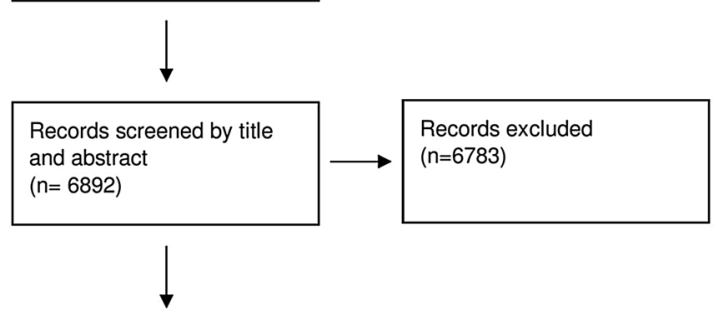

Full text articles assessed for eligibility $(n=109)$

$\longrightarrow \quad$ Full-text articles excluded because 1) It didn't report functional outcome $(n=30)$

2) It didn't have own data $(n=18)$

3) No dissection of the rectum reported $(n=3)$

4) Ongoing study $(n=2)$

5) Others $(n=5)$

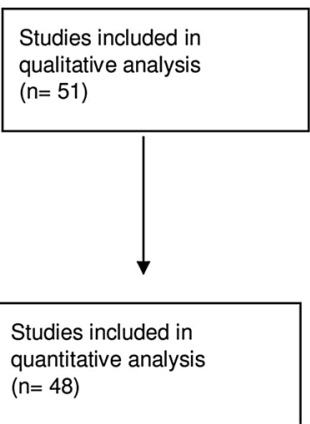

Excluded because data could not be pooled. approach in non-RCTs (odds ratio (OR) [95\% confidence interval $(\mathrm{CI})] 0.86[0.75,0.98])$, while there were no differences for RCTs (OR [CI] 0.88 [0.33, 1.93]). The heterogeneity was low in both, overall and subgroup analyses $\left(I^{2}=0 \%\right)$ (Fig. 2).

\subsubsection{Urinary retention}

Furthermore, for urinary retention (19 non-RCTs, 1 RCT, 4535 patients), there was a significant reduction in urinary retention rate for patients that received RAS compared to laparoscopic in non-RCTs (OR (CI)] 0.65 [0.46, 0.92]). Only one RCT reported on did no show significant differences 


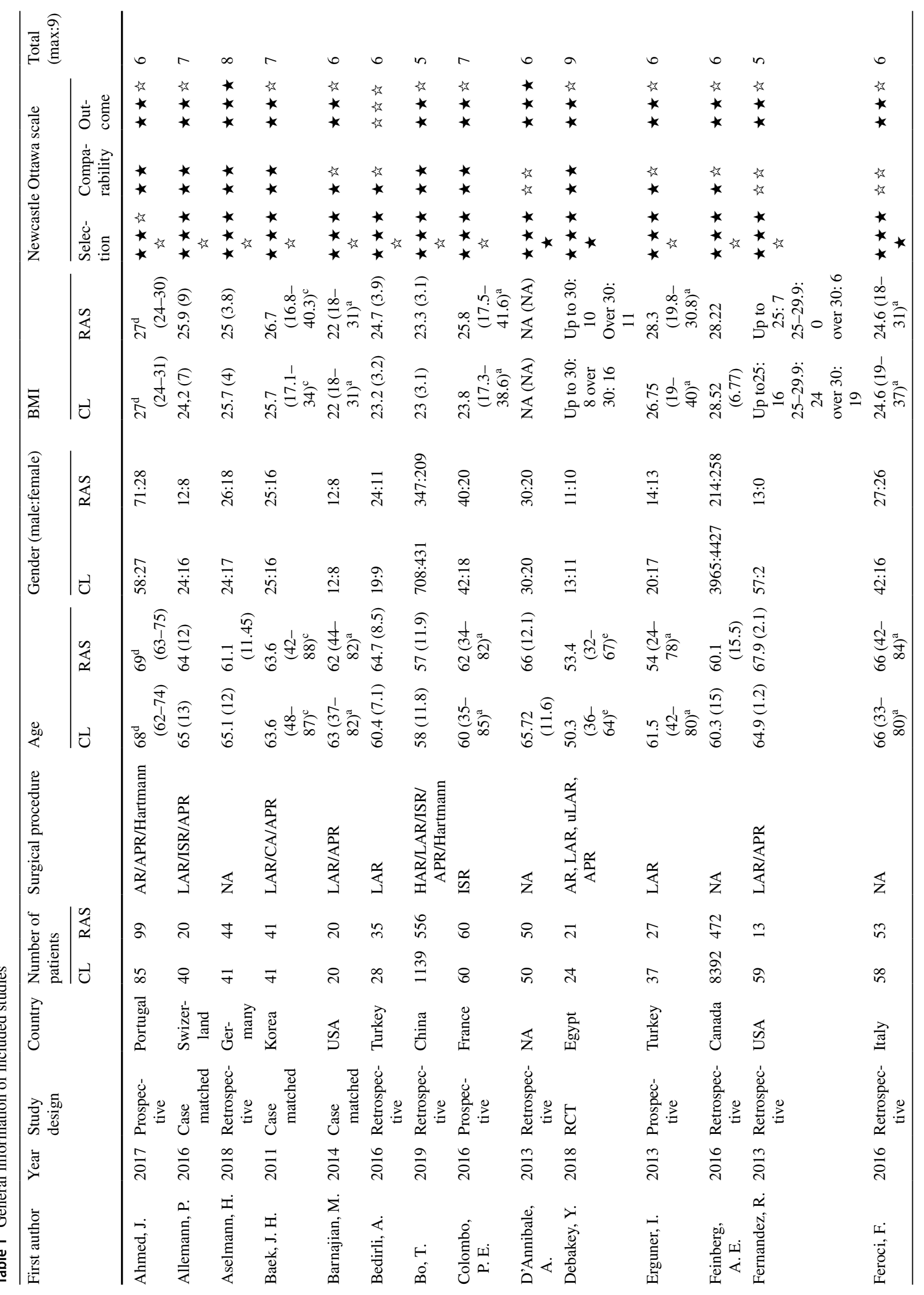




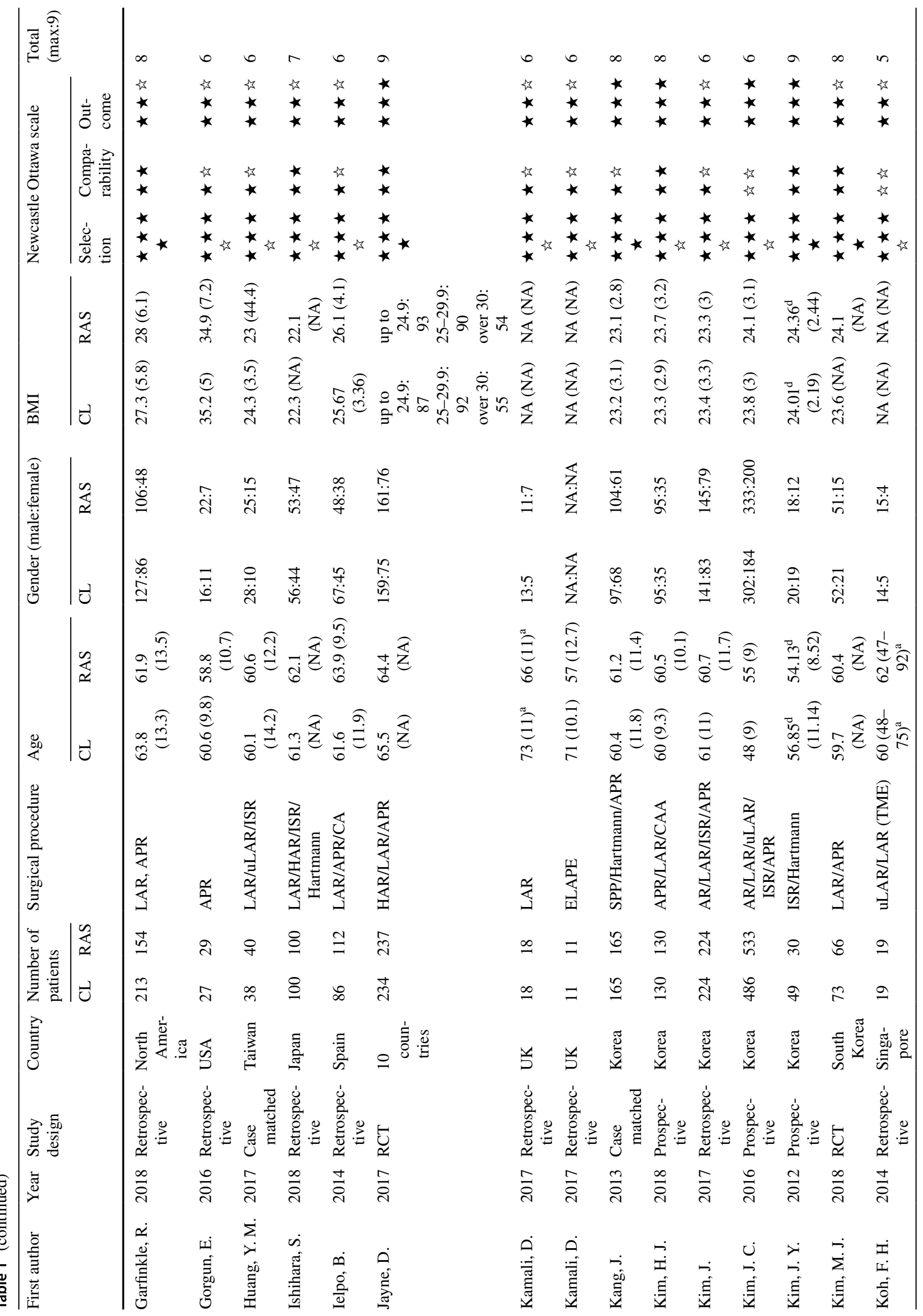




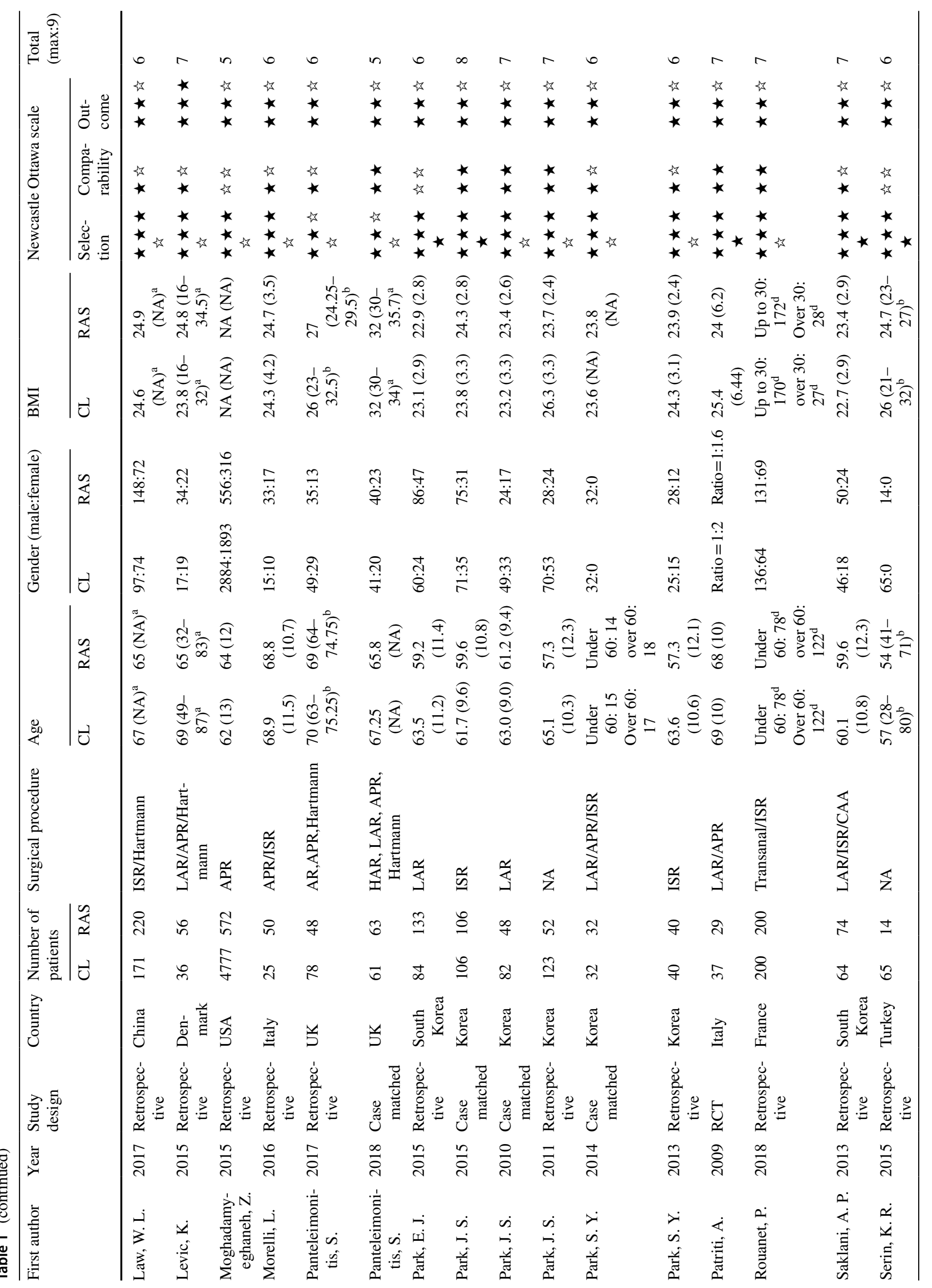




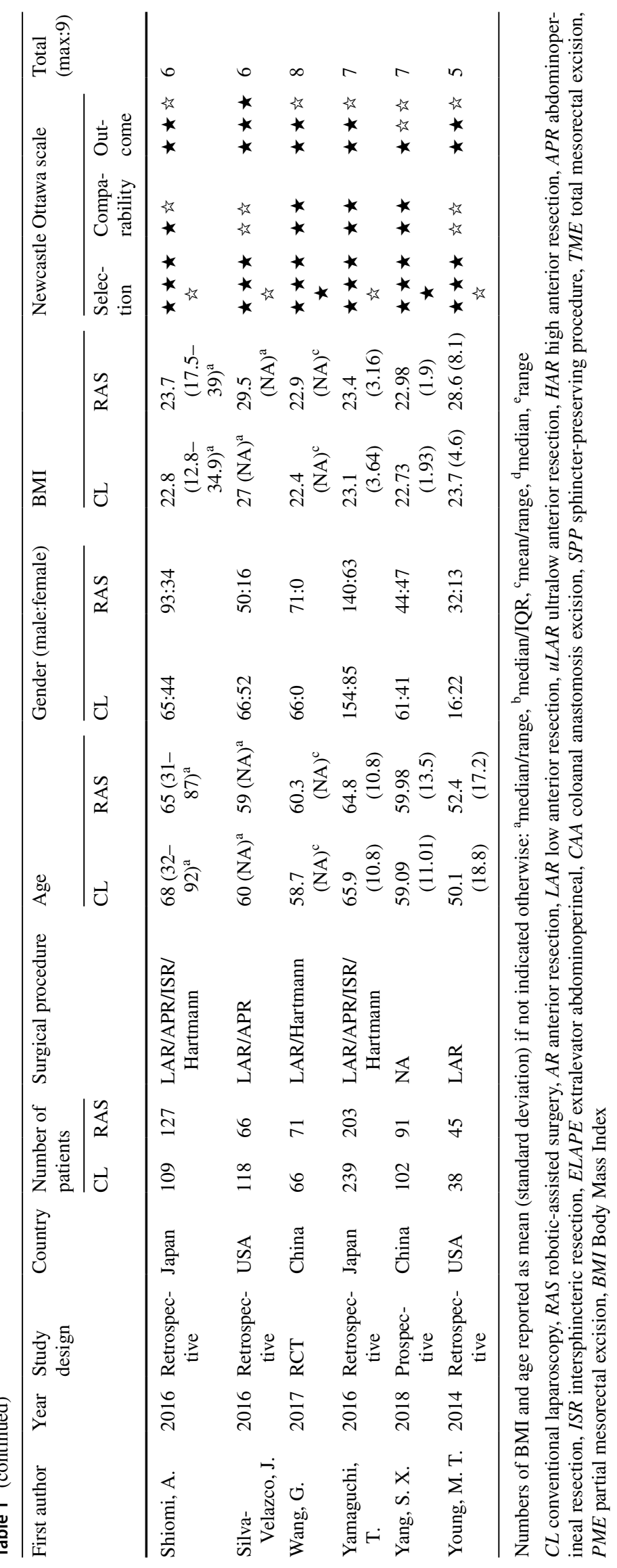




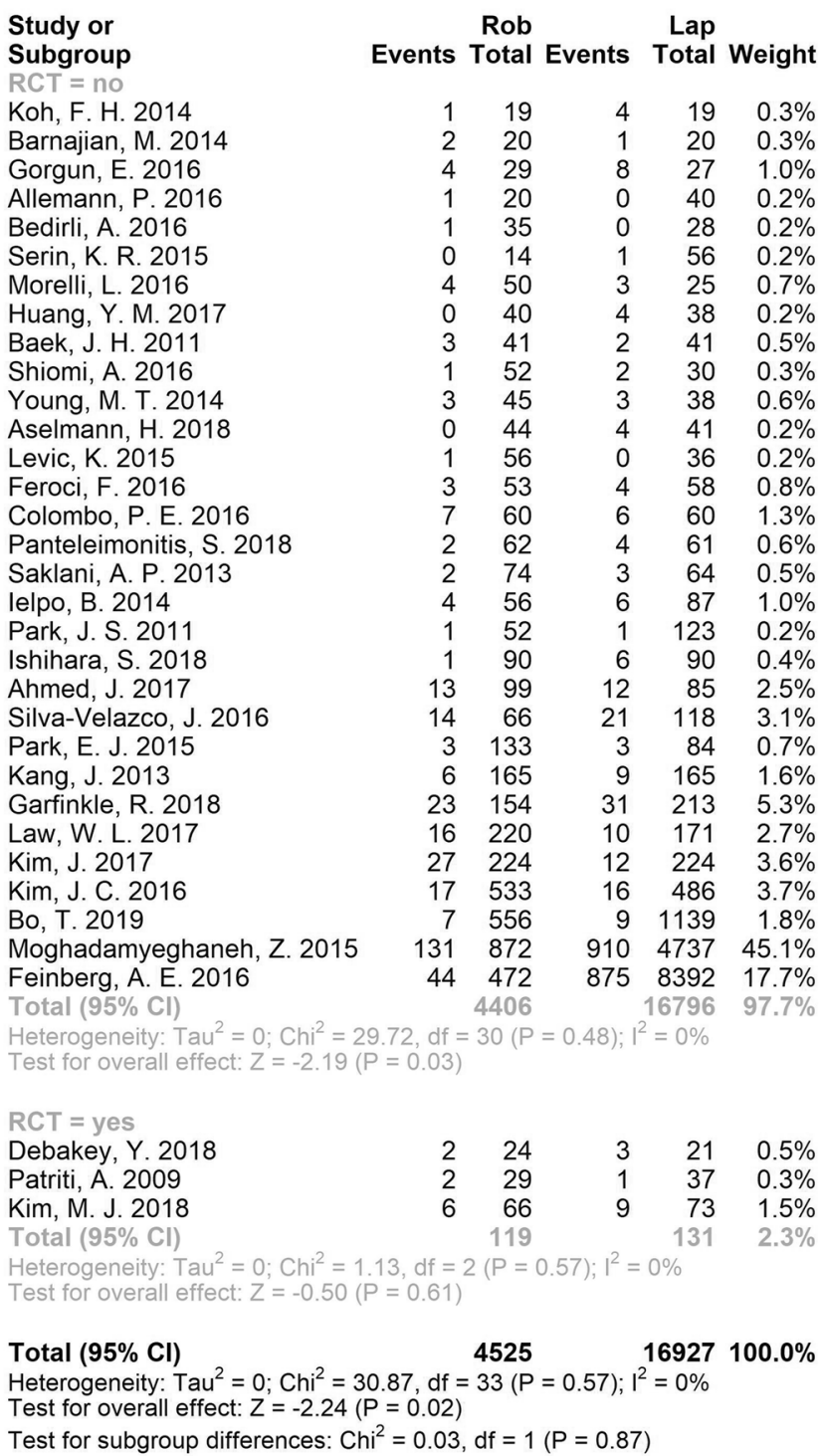

Odds Ratio

, Random, 95\% CI

0.21 [0.02; 2.07]

$2.11[0.18 ; 25.35]$

$0.38[0.10 ; 1.45]$

$6.23[0.24 ; 160.03]$

$2.48[0.10 ; 63.21]$

$1.28[0.05 ; 32.98]$

$0.64[0.13 ; 3.10]$

$0.09[0.00 ; 1.82]$

$1.54[0.24 ; 9.73]$

$0.27[0.02 ; 3.16]$

$0.83[0.16 ; 4.39]$

$0.09[0.00 ; 1.80]$

$1.97[0.08 ; 49.76]$

$0.81[0.17 ; 3.80]$

$1.19[0.37 ; 3.77]$

$0.48[0.08 ; 2.69]$

$0.56[0.09 ; 3.49]$

$1.04[0.28 ; 3.86]$

$2.39[0.15 ; 38.99]$

$0.16[0.02 ; 1.33]$

$0.92[0.40 ; 2.14$

$1.24[0.58 ; 2.65]$

$0.62[0.12 ; 3.16]$

$0.65[0.23 ; 1.88]$

$1.03[0.57 ; 1.85]$

$1.26[0.56 ; 2.86]$

$2.42[1.19 ; 4.91]$

$0.97[0.48 ; 1.94]$

$1.60[0.59 ; 4.32]$

$0.74[0.61 ; 0.91]$

$0.88[0.64 ; 1.21]$

$0.86[0.75 ; 0.98]$

$0.55[0.08 ; 3.63]$

$2.67[0.23 ; 30.96]$

$0.71[0.24 ; 2.12]$

$0.80[0.33 ; 1.93]$

$0.86[0.75 ; 0.98]$

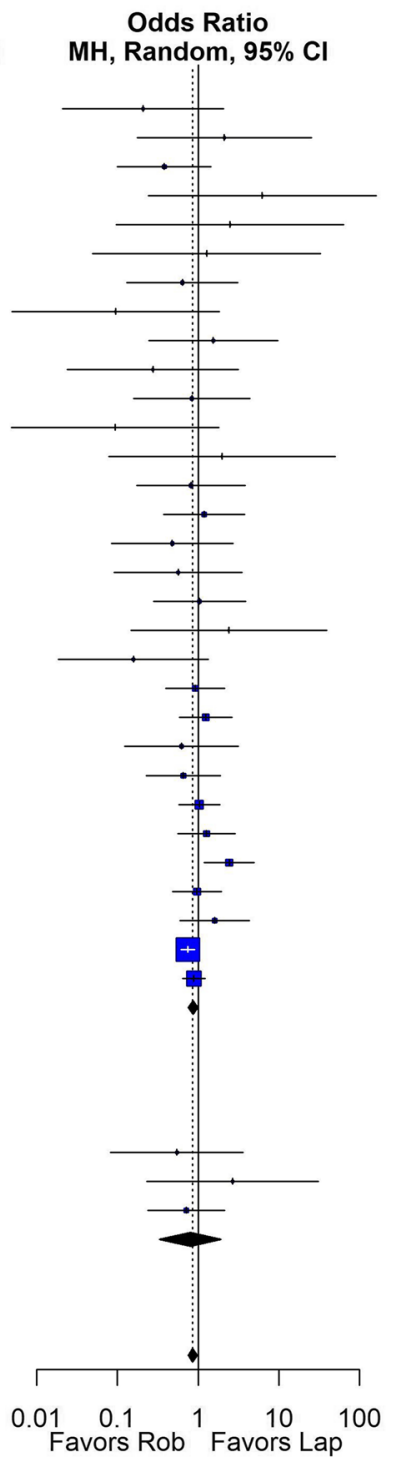

Fig. 2 Pooled analysis for ileus

(OR (CI)] 1.29 [0.08, 21.47]). The heterogeneity was low for both, overall analysis and non-RCTs $\left(I^{2}=0 \%\right)$ (Fig. 3).

\subsubsection{Sexual function}

Total IIEF scores were reported in five studies ( 3 non-RCTs, 2 RCTs, 512 patients). There was no difference for RCTs (standardized mean difference (SDM) [CI] 0.09 [- 0.14, $0.31]$ ) or non-RCTs (SMD (CI)] 0.46 [- 0.13, 1.04]) was noted. Heterogeneity was moderate for overall comparison $\left(I^{2}=57 \%\right)$, high for non-RCTs $\left(I^{2}=72 \%\right)$, while it was absent for RCTs $\left(I^{2}=0 \%\right)$ (Fig. $\left.4 \mathrm{~A}\right)$.

Additionally, Kim et al. reported sexual functioning with the QLQ-CR 38 and found a better sexual function after 12-month for the RAS group (RAS mean [CI] 35.2
[26.9, 43.5] versus laparoscopy mean [CI] 23.0 [15.7, 30.2], $p=0.032$ ) [34]. Furthermore, Jayne et al. assessed female sexual functioning with the Female Sexual Function Index (FSFI, higher scores indicating better function) and found no significant difference between scores for the laparoscopic and the RAS group at 6-month postoperatively (laparoscopy minus RAS: 1.231 ; CI $-3.54,6.00 ; p=0.60)$ [11]. Rouanet et al. did not find significant differences for the FSFI or IIEF between RAS and laparoscopy but provided graphical results which were not feasible for pooling [35].

\subsubsection{Urinary symptoms}

In order to assess urinary symptoms, the IPSS was used by a total of 7 studies ( 5 non-RCTs, 2 RCTs, 1010 patients). The 


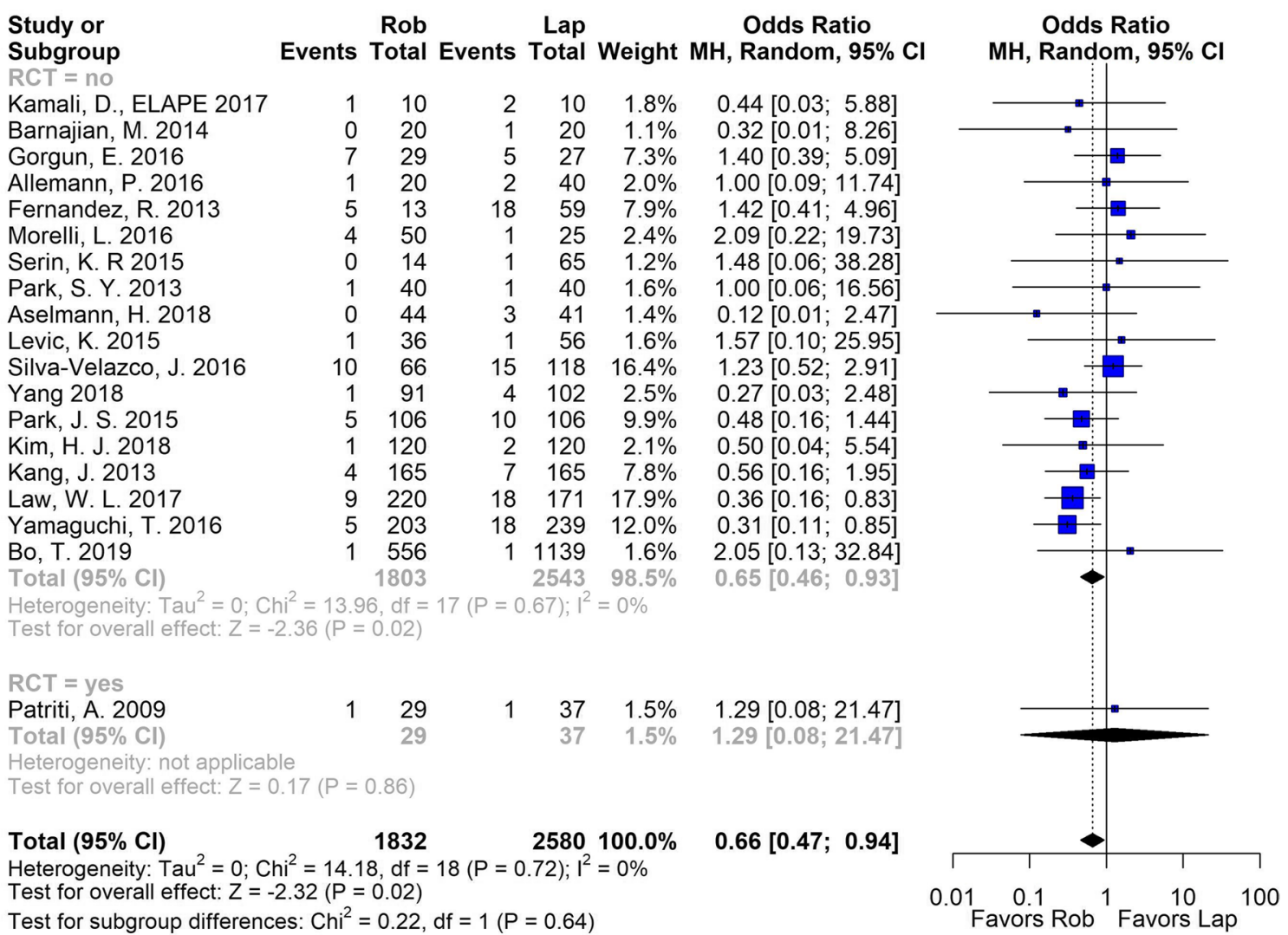

Fig. 3 Pooled analysis for urinary retention

advantage of RAS was statistically significant for non-RCTs (MD [CI] - 0.60 [- 1.17, - 0.03]), while no more differences were found in RCTs (MD [CI] - 1.37 [-4.18, 1.44]). Accordingly, heterogeneity was high in RCTs $\left(I^{2}=81 \%\right)$, absent in non-RCTs $\left(\mathrm{I}^{2}=0 \%\right)$ and low in their combined analysis $\left[I^{2}=13\right.$ (Fig. 4B)].

\subsubsection{Quality of life}

Three studies (all non-RCTs; 308 patients) reported usable data on quality of life using the core quality of life questionnaire (QLQ-C30). Patients who underwent RAS had a significantly higher score at different points in time after surgery compared to patients who underwent laparoscopy (MD [CI] 2.99 [2.02, 3.95], $p<0.001$ ) (Fig. 4C). The heterogeneity was low $\left(I^{2}=0 \%\right)$. In addition, Rouanet et al. also found no differences between the two groups [35]. Kim et al. reported no differences between laparoscopy and RAS for quality of life at 3 weeks, 3 months and 12 months [34].

\subsubsection{Quality of evidence and bias assessment}

As only four of the included studies were RCTs, all studies were assessed with the NOS in order to ensure comparability. The included studies were of heterogeneous quality (Table 1). Consequently, the certainty in evidence according to GRADE was rated as moderate to very low (Supplementary Material). Funnel plots were used to assess potential publication bias. However, none of the funnel plots raised concerns for publication bias (Supplementary Material-Statistical Analysis).

\section{Discussion}

This systematic review and meta-analysis summarizes the existing evidence in terms of functional outcomes after robotic-assisted or laparoscopic rectal resection for cancer. Overall the results show advantages in favor of the robotic approach regarding ileus, urinary retention, urinary function, and quality of life, while there were no differences concerning sexual functioning.

When compared to open surgery, laparoscopy has shown to provide potential advantages during the perioperative period such as a faster return of bowel function [36] and a shorter hospital stay after rectal cancer surgery [5]. However, no consistent advantages regarding ileus were recorded in RCTs comparing laparoscopy to open surgery [36]. 


\section{A Erectile function}

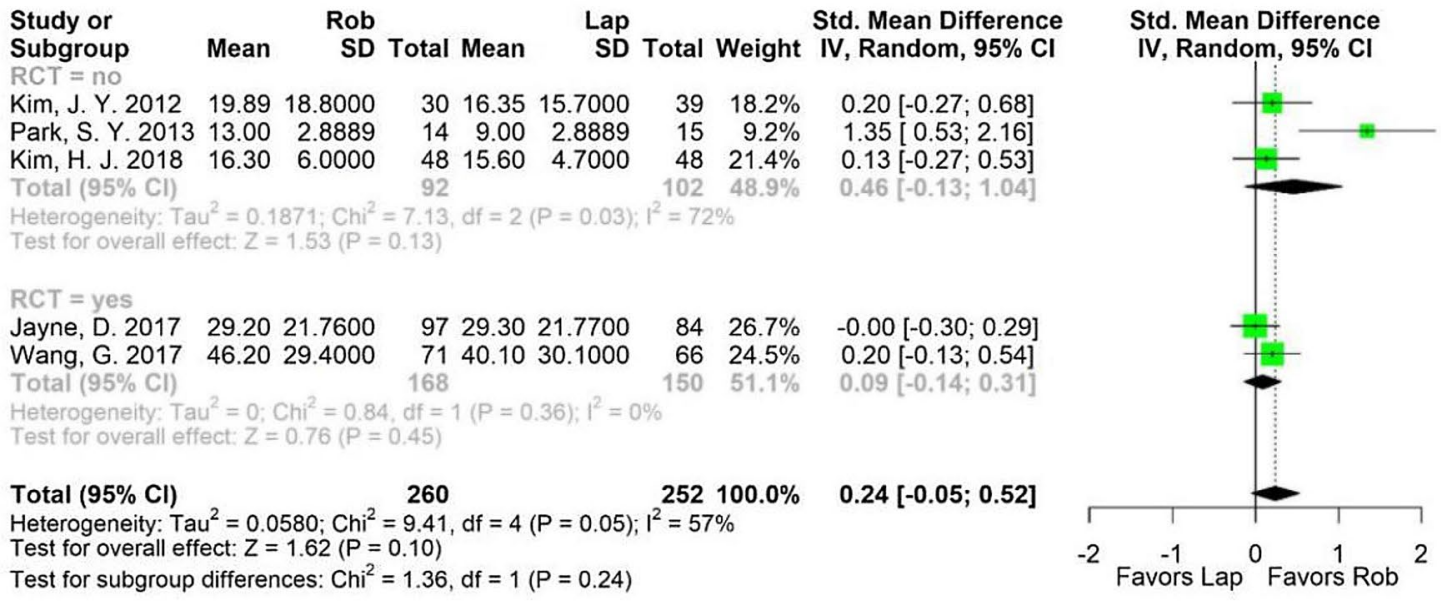

\section{B Urinary function}

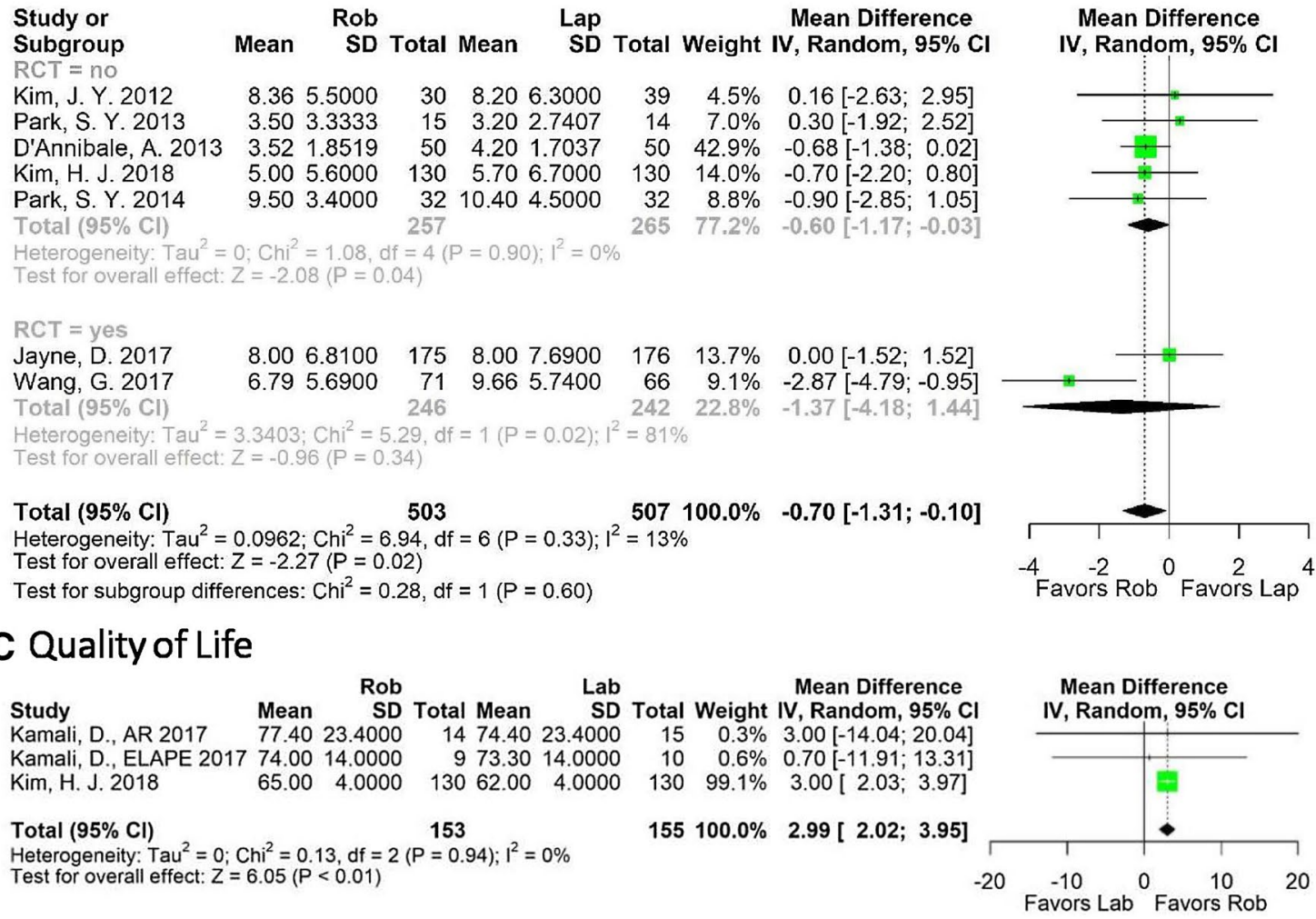

Fig. 4 Pooled analysis for $\mathbf{A}$ erectile function; $\mathbf{B}$ urinary function; $\mathbf{C}$ quality of life

Conversely, the present pooled analysis provides evidence that RAS may help reduce the rate of postoperative ileus compared to CL.

Urinary retention which requires prolonged catheterization was also significantly reduced for RAS compared to CL in the present analysis.

Mid- and long-term outcomes such as QoL and sexual, urinary and bowel functioning are very relevant to patients after the initial postoperative period. Despite the small number of included studies for this outcome, RAS was associated with improved QoL. The most prevalent assessment score used in the included studies was the QLQ C-30 which was developed by the European Organization for Research and Treatment of Cancer (EORTC) and is most commonly used to measure the QoL for cancer patients [37, 38]. Yet, systematic assessment of QoL has been underrepresented in 
surgical practice and research. In order to capture all facets of a disease and its impact on QoL, "patient reported outcomes" are gaining widespread acceptance. These are an effective approach to comprehensively collecting patients perception of the underlying disease [39]. Therefore, these measurements should be incorporated into the clinical pathway and especially into surgical trials, in order to gain more insight into the impact of different treatment approaches on QoL and to confirm the hypotheses generated by the presented study.

In terms of urinary and erectile functioning it was noticeable that the outcome measures were mainly scores that were not primarily designed to evaluate patients after rectal surgery. The IPSS was first introduced into urology by the American Urological Association in order to assess patient perception of problems derived from benign prostatic hyperplasia [40]. Therefore, it remains questionable whether this score is the optimal choice seeing as symptoms such as straining or weak stream could still be attributed to obstructive symptoms due to an enlarged prostate. Regarding these limitations, studies should check for potential baseline differences in different age groups and stratified randomization based on baseline characteristics should be considered. Additionally, comparability between sexes is not possible as women obviously require different scores.

Similar considerations can be made when assessing sexual function. For example, the ROLARR trial used the IIEF and FSFI to assess sexual function [11]. Despite the high quality of the trial, only $57 \%$ of men and $36 \%$ of women completed the assessment scores which underlines the low emphasis and neglect of the relevance of functional outcomes in current trials. Additionally, less than $20 \%$ of the included studies reported erectile or sexual function at all. Moreover, while potential advantages regarding ileus, urinary retention rates, urinary symptoms as well as QoL for RAS over CL were found, no differences were found for sexual function. In consequence, in the present study it appears that RAS potentially provides better functional outcomes compared to CL.

Seen from a technical perspective, reasons for improved outcomes of RAS may include better visualization due to $3 \mathrm{D}$ vision, thus possibly enabling improved detection of risk structures and more accurate dissection due to the stable instrument platform and precise instrumentation. In addition, RAS seems to unfold its potential with more complex procedures. Hence for procedures with high complexity during the reconstruction phase such as pancreaticoduodenectomy or esophagectomy, the laparoscopic approach has failed to gain widespread acceptance due to its technical difficulty. Therefore, these procedures are mainly performed in specialized centers. For example, the recent Dutch Leopard-II study was terminated early due to increased mortality in the laparoscopic group [41]. A subsequent meta-analysis comparing open and laparoscopic pancreaticoduodenectomy including only RCTs did not find any relevant advantage which would support the use of laparoscopy for this procedure [42, 43]. However, the robotic approach to pancreaticoduodenectomy seems to offer a promising alternative to the open procedure with comparable postoperative outcomes and low mortality and should thus be evaluated in RCTs [12].

However, as controversially discussed, the surgeon herself or himself may be a main contributor to the surgical outcome. While there will always be interpersonal differences between surgeons which can hardly be eliminated, each individual has to go through a considerable learning curve. The presence of learning effects is well acknowledged in the surgical community and structured training programs have been introduced. However, it has been shown that previous experience in open or laparoscopic surgery does no translate into better performance with RAS [44]. With this in mind, the learning curve of RAS for rectal cancer takes approximately 20 to 35 cases [45-47]. In comparison, the learning curve of laparoscopic colorectal surgery has been shown to be between 80 and 150 cases depending on the outcome parameter in a systematic review and international multicenter analysis [48]. Of the included studies in this manuscript, a considerable amount reported on their initial experience with RAS without adjusting for learning effects. An excellent example of accounting for surgeon experience is the ROLARR trial by Jayne et al. [11]. Here it was clearly defined that participating surgeons had to have performed at least 30 minimally invasive rectal cancer resections with at least 10 robotic and 10 laparoscopic procedures. As a matter of fact, the ROLARR trial served as real-world example in a consecutive study by Corrigan et al. that accounted for experience of participating surgeons [49]. While the original paper reported an OR of 0.61 (CI $0.31,1.21 ; p=0.16$ ) for the primary endpoint (conversion), the study by Corrigan et al. found that patients who were operated by surgeons with the mean experience of all ROLARR surgeons (153 laparoscopic cases; 68 robotic cases), had a lower OR of 0.40 favoring the robotic approach (CI $0.168,0.953 ; p=0.039$ ). Furthermore, for surgeons who had performed at least 100 robotic procedures, the odds of conversion were significantly lower with the robotic approach, independent from the number of laparoscopic procedures performed (i.e. 45, 91 or 180 cases). Overall there are learning effects for both approaches, RAS and laparoscopy, while RAS seems to be easier to adopt, thus less cases (and patients) are needed to optimize quality of surgical care.

Finally, since minimally invasive techniques are more often used, an emerging third option besides RAS and traditional laparoscopy is transanal TME (taTME). taTME offers some potential advantages but also seems to have some drawbacks especially in terms of implementation and learning curves. The available studies on this technique 
showed contradicting results, hence it remains to be further investigated according to IDEAL stages [50, 51]. In accordance with the previous paragraph stringent patient selection, dedicated training, and high case-volume in specialized centers should be integrated to avoid adverse outcomes [52].

\section{Limitations}

The major limitations of the present systematic review and meta-analysis are the heterogeneous reporting measures for the respective studies. For example, there is an original and a simplified version of the IIEF-5 which were both used in different studies. Further limitations include the use of only aggregated data as there was no access to primary data sources and no stratification by surgical approach (e.g., high and low anterior resection, Hartmann's procedure, intersphincteric resection). For future trials it would thus be desirable to distinguish between the different procedures since this could influence functional outcomes. However, appropriate statistical measures were applied to account for these variations and all analyses were performed by a senior statistician. Additionally, the studies were not powered for functional outcomes as primary endpoints which might reduce the trust in the presented results.

Furthermore, there was no consistent definition of the operative approach in terms of clear differentiation. For example, between LAR and HAR. Both anatomical landmarks, such as above/below the peritoneal flap as well as distances from the anus in centimeter were reported. The limited quality of the included studies itself resulting from mostly non-randomized studies should not be considered as a limitation, since quality assessment is a crucial step of each systematic review which helps to understand drawbacks of recent studies.

\section{Implications for future research}

As discussed previously, the current evidence remains equivocal but this meta-analysis summarizes the existing data, identifies gaps of the current evidence and thus can serve as a basis for generating hypotheses and performing sample size calculations of further studies with functional outcome as primary outcome. In addition, the term functional outcomes must be further defined and clarified. For example, some scores were adopted from other specialties such as urology and might fail to assess further symptoms that are specific to rectal surgery. Along with this, patient characteristics and subgroups (body mass index, gender, previous surgery or neoadjuvant treatment) should be taken into consideration in order to provide patients with a more individualized treatment. Finally, full analysis for the learning curve and differences in tumor stages as well as the different procedures should be further investigated.

\section{Conclusions}

The current systematic review and meta-analysis suggests that there are potential benefits for robotic-assisted surgery over traditional laparoscopy in terms of functional outcomes after rectal cancer resection in non-randomized trials. The current evidence is limited due to a lack of RCTs and the reporting of functional outcomes as secondary endpoints. While robotic-assisted surgery has proven safe with regard to oncological endpoints, there is a need for high-quality randomized-controlled trials which are adequately powered for functional outcomes (e.g., urinary function, sexual function, quality of life).

Acknowledgements Open Access funding provided by Projekt DEAL. We would like to thank Mr. Dietmar Fleischer from Heidelberg's University Library for conducting the literature search. As scholar of the Research Talent Academy of the European Association of Endoscopic Surgery, Karl-Friedrich Kowalewski received methodological support during the conduct of this study.

\section{Compliance with ethical standards}

Disclosures Felix Nickel reports receiving travel support for conference participation as well as equipment provided for laparoscopic surgery courses by KARL STORZ, Johnson \& Johnson, Intuitive and Medtronic. Yakub Kulu has received travel and robotic surgery training support as well as financial support for conference participation by Inutituve Surgical and Medtronic. Karl-Friedrich Kowalewski, Laura Seifert, Seher Ali, Caelan Max Haney, Mona Schmidt, Svenja Seide, Christian Tapking, Andreas Shamiyeh, Thilo Hackert and Beat MüllerStich have no conflicts of interest or financial ties to disclose.

Open Access This article is licensed under a Creative Commons Attribution 4.0 International License, which permits use, sharing, adaptation, distribution and reproduction in any medium or format, as long as you give appropriate credit to the original author(s) and the source, provide a link to the Creative Commons licence, and indicate if changes were made. The images or other third party material in this article are included in the article's Creative Commons licence, unless indicated otherwise in a credit line to the material. If material is not included in the article's Creative Commons licence and your intended use is not permitted by statutory regulation or exceeds the permitted use, you will need to obtain permission directly from the copyright holder. To view a copy of this licence, visit http://creativecommons.org/licenses/by/4.0/.

\section{Appendix}

PubMed search strategy

(Rectal Cancer [Title/Abstract] OR Rectum Cancer* [Title/Abstract] OR Rectal Tumor* [Title/Abstract] OR Rectum Neoplasm* [Title/Abstract] OR Total mesorectal exicision [Title/Abstract] OR tme [Title/Abstract] OR rectum [Title/Abstract] OR mesorectal [Title/Abstract] OR Rectal Neoplasms [mesh] OR Rectum/surgery [mesh]). 


\section{AND}

(laparoscopic [Title/Abstract] OR laporoscopy [Title/ Abstract] OR minimal access surgery [Title/Abstract] OR minimally access [Title/Abstract] OR "minimally invasive" [Title/Abstract]OR Laparoscopy [mesh] OR Minimally Invasive Surgical Procedures [mesh]).

\section{AND}

(Robotic [Title/Abstract] OR robotic surgery [Title/ Abstract] OR robotic-assisted surgery [Title/Abstract] OR robot-assisted surgery [Title/Abstract] OR robotic-assisted [Title/Abstract] OR robotic [Title/Abstract] OR robotassisted [Title/Abstract] OR robot assisted [Title/Abstract] OR da vinci [Title/Abstract] OR da vinci [Title/Abstract] OR Robotic Surgical Procedures [mesh] OR Surgical Procedures, Operative [mesh] OR Minimally Invasive Surgical Procedures [mesh]).

\section{References}

1. Siegel RL, Miller KD, Fedewa SA, Ahnen DJ, Meester RG, Barzi A et al (2017) Colorectal cancer statistics, 2017. CA Cancer J Clin 67(3):177-193

2. Heald RJ, Moran BJ, Ryall RH, Sexton R, MacFarlane JK (1998) Rectal cancer: the basingstoke experience of total mesorectal excision, 1978-1997. Arch Surg 133(8):894-898

3. Heald R, Husband E, Ryall R (1982) The mesorectum in rectal cancer surgery-the clue to pelvic recurrence? Br J Surg 69(10):613-616

4. Glynne-Jones R, Wyrwicz L, Tiret E, Brown G, Rödel C, Cervantes A et al (2017) Rectal cancer: ESMO Clinical Practice Guidelines for diagnosis, treatment and follow-up. Ann Oncol 28(suppl 4):22-40

5. Bonjer HJ, Deijen CL, Abis GA, Cuesta MA, van der Pas MH, de Lange-de Klerk ES et al (2015) A randomized trial of laparoscopic versus open surgery for rectal cancer. N Engl J Med 372(14):1324-1332

6. Jeong S-Y, Park JW, Nam BH, Kim S, Kang S-B, Lim S-B et al (2014) Open versus laparoscopic surgery for mid-rectal or lowrectal cancer after neoadjuvant chemoradiotherapy (COREAN trial): survival outcomes of an open-label, non-inferiority, randomised controlled trial. Lancet Oncol 15(7):767-774

7. Kang S-B, Park JW, Jeong S-Y, Nam BH, Choi HS, Kim D-W et al (2010) Open versus laparoscopic surgery for mid or low rectal cancer after neoadjuvant chemoradiotherapy (COREAN trial): short-term outcomes of an open-label randomised controlled trial. Lancet Oncol 11(7):637-645

8. Stevenson AR, Solomon MJ, Lumley JW, Hewett P, Clouston AD, Gebski VJ et al (2015) Effect of laparoscopic-assisted resection vs open resection on pathological outcomes in rectal cancer: the ALaCaRT randomized clinical trial. JAMA 314(13):1356-1363

9. Fleshman J, Branda M, Sargent DJ, Boller AM, George V, Abbas M et al (2015) Effect of laparoscopic-assisted resection vs open resection of stage II or III rectal cancer on pathologic outcomes: the ACOSOG Z6051 randomized clinical trial. JAMA 314(13):1346-1355

10. Nienhuser H, Heger P, Schmitz R, Kulu Y, Diener MK, Klose J et al (2018) Short- and long-term oncological outcome after rectal cancer surgery: a systematic review and meta-analysis comparing open versus laparoscopic rectal cancer surgery. J Gastrointest Surg 22(8):1418-1433
11. Jayne D, Pigazzi A, Marshall H, Croft J, Corrigan N, Copeland J et al (2017) Effect of robotic-assisted vs conventional laparoscopic surgery on risk of conversion to open laparotomy among patients undergoing resection for rectal cancer: the ROLARR randomized clinical trial. JAMA 318(16):1569-1580

12. Zureikat AH, Moser AJ, Boone BA, Bartlett DL, Zenati M, Zeh HJ (2013) 250 robotic pancreatic resections: safety and feasibility. Ann Surg 258(4):554-562

13. Van der Sluis P, Ruurda J, Verhage R, van der Horst S, Haverkamp L, Siersema P et al (2015) Oncologic long-term results of robotassisted minimally invasive thoraco-laparoscopic esophagectomy with two-field lymphadenectomy for esophageal cancer. Ann Surg Oncol 22(3):1350-1356

14. Prete FP, Pezzolla A, Prete F, Testini M, Marzaioli R, Patriti A et al (2018) Robotic versus laparoscopic minimally invasive surgery for rectal cancer: a systematic review and meta-analysis of randomized controlled trials. Ann Surg 267(6):1034-1046

15. Simillis C, Lal N, Thoukididou SN, Kontovounisios C, Smith JJ, Hompes R et al (2019) Open versus laparoscopic versus robotic versus transanal mesorectal excision for rectal cancer: a systematic review and network meta-analysis. Ann Surg 270(1):59-68

16. Edwards BK, Ward E, Kohler BA, Eheman C, Zauber AG, Anderson RN et al (2010) Annual report to the nation on the status of cancer, 1975-2006, featuring colorectal cancer trends and impact of interventions (risk factors, screening, and treatment) to reduce future rates. Cancer 116(3):544-573

17. Haggar FA, Boushey RP (2009) Colorectal cancer epidemiology: incidence, mortality, survival, and risk factors. Clin Colon Rectal Surg 22(4):191

18. Siegel R, DeSantis C, Jemal A (2014) Colorectal cancer statistics, 2014. CA Cancer J Clin 64(2):104-117

19. Novara G, Ficarra V, Mocellin S, Ahlering TE, Carroll PR, Graefen $M$ et al (2012) Systematic review and meta-analysis of studies reporting oncologic outcome after robot-assisted radical prostatectomy. Eur Urol 62(3):382-404

20. Holmer C, Kreis ME (2018) Systematic review of robotic low anterior resection for rectal cancer. Surg Endosc 32:569-581

21. Guerra F, Pesi B, Amore Bonapasta S, Perna F, Di Marino M, Annecchiarico $\mathrm{M}$ et al (2016) Does robotics improve minimally invasive rectal surgery? Functional and oncological implications. J Dig Dis 17(2):88-94

22. Moher D, Liberati A, Tetzlaff J, Altman DG (2009) Preferred reporting items for systematic reviews. Ann Intern Med 151(4):264-269

23. Shea BJ, Reeves BC, Wells G, Thuku M, Hamel C, Moran J et al (2017) AMSTAR 2: a critical appraisal tool for systematic reviews that include randomised or non-randomised studies of healthcare interventions, or both. BMJ 358:j4008

24. Goossen K, Tenckhoff S, Probst P, Grummich K, Mihaljevic AL, Büchler MW et al (2018) Optimal literature search for systematic reviews in surgery. Langenbeck Arch Surg 403(1):119-129

25. Santos CMDC, Pimenta CADM, Nobre MRC (2007) The PICO strategy for the research question construction and evidence search. Rev Lat Am Enfermagem 15(3):508-511

26. Weber PA, Merola S, Wasielewski A, Ballantyne GH (2002) Telerobotic-assisted laparoscopic right and sigmoid colectomies for benign disease. Dis Colon Rectum 45(12):1689-1694 (Discussion 95-96)

27. Wells G (2001) The Newcastle-Ottawa SCALE (NOS) for assessing the quality of non randomised studies in meta-analyses. https ://www.ohri.ca/programs/clinical_epidemiology/oxford.asp

28. Guyatt GH, Oxman AD, Vist GE, Kunz R, Falck-Ytter Y, AlonsoCoello P et al (2008) Rating quality of evidence and strength of recommendations: GRADE: an emerging consensus on rating quality of evidence and strength of recommendations. BMJ 336(7650):924 
29. Mantel N, Haenszel W (1959) Statistical aspects of the analysis of data from retrospective studies of disease. J Natl Cancer Inst 22(4):719-748

30. Greenland S, Robins JM (1985) Sparse follow-up data. Biometrics 41(1):55-68

31. Higgins JP, Green S (2011) Cochrane handbook for systematic reviews of interventions. Wiley, Hoboken

32. Team RC (2013) R: a language and environment for statistical computing. RC Team, Vienna

33. Schwarzer G (2007) Meta: an R package for meta-analysis. $R$ News 7(3):40-45

34. Kim MJ, Park SC, Park JW, Chang HJ, Kim DY, Nam BH et al (2018) Robot-assisted versus laparoscopic surgery for rectal cancer: a phase II open label prospective randomized controlled trial. Ann Surg 267(2):243-251

35. Rouanet P, Bertrand MM, Jarlier M, Mourregot A, Traore D, Taoum C et al (2018) Robotic versus laparoscopic total mesorectal excision for sphincter-saving surgery: results of a single-center series of 400 consecutive patients and perspectives. Ann Surg Oncol 25(12):3572-3579

36. van der Pas MH, Haglind E, Cuesta MA, Fürst A, Lacy AM, Hop WC et al (2013) Laparoscopic versus open surgery for rectal cancer (COLOR II): short-term outcomes of a randomised, phase 3 trial. Lancet Oncol 14(3):210-218

37. McKenzie L, Van Der Pol M (2009) Mapping the EORTC QLQ C-30 onto the EQ-5D instrument: the potential to estimate QALYs without generic preference data. Value Health 12(1):167-171

38. Aaronson NK, Ahmedzai S, Bergman B, Bullinger M, Cull A, Duez NJ et al (1993) The European Organization for Research and Treatment of Cancer QLQ-C30: a quality-of-life instrument for use in international clinical trials in oncology. JNCI 85(5):365-376

39. Black N (2013) Patient reported outcome measures could help transform healthcare. BMJ 346:f167

40. Barry MJ, Fowler FJ, O’Leary MP, Bruskewitz RC, Holtgrewe HL, Mebust WK et al (1992) The American Urological Association symptom index for benign prostatic hyperplasia. J Urol 148(51):1549-1557

41. van Hilst J, de Rooij T, Bosscha K, Brinkman DJ, van Dieren S, Dijkgraaf MG et al (2019) Laparoscopic versus open pancreatoduodenectomy for pancreatic or periampullary tumours (LEOPARD-2): a multicentre, patient-blinded, randomised controlled phase 2/3 trial. Lancet Gastroenterol Hepatol 4(3):199-207
42. Nickel F, Haney CM, Kowalewski KF, Probst P, Limen EF, Kalkum E et al (2019) Laparoscopic versus open pancreaticoduodenectomy: a systematic review and meta-analysis of randomized controlled trials. Ann Surg 271:54-66

43. Strobel O, Buchler MW (2019) Laparoscopic pancreatoduodenectomy: safety concerns and no benefits. Lancet Gastroenterol Hepatol 4(3):186-187

44. Kowalewski KF, Schmidt MW, Proctor T, Pohl M, Wennberg E, Karadza E et al (2018) Skills in minimally invasive and open surgery show limited transferability to robotic surgery: results from a prospective study. Surg Endosc 32:1656-1667

45. Bokhari MB, Patel CB, Ramos-Valadez DI, Ragupathi M, Haas EM (2011) Learning curve for robotic-assisted laparoscopic colorectal surgery. Surg Endosc 25(3):855-860

46. Spinoglio G, Summa M, Priora F, Quarati R, Testa S (2008) Robotic colorectal surgery: first 50 cases experience. Dis Colon Rectum 51(11):1627-1632

47. Sng KK, Hara M, Shin J-W, Yoo B-E, Yang K-S, Kim S-H (2013) The multiphasic learning curve for robot-assisted rectal surgery. Surg Endosc 27(9):3297-3307

48. Miskovic D, Ni M, Wyles SM, Tekkis P, Hanna GB (2012) Learning curve and case selection in laparoscopic colorectal surgery: systematic review and international multicenter analysis of 4852 cases. Dis Colon Rectum 55(12):1300-1310

49. Corrigan N, Marshall H, Croft J, Copeland J, Jayne D, Brown $\mathrm{J}$ (2018) Exploring and adjusting for potential learning effects in ROLARR: a randomised controlled trial comparing roboticassisted vs. standard laparoscopic surgery for rectal cancer resection. Trials 19(1):339

50. Hol JC, van Oostendorp SE, Tuynman JB, Sietses C (2019) Longterm oncological results after transanal total mesorectal excision for rectal carcinoma. Tech Coloproctol 23:903-911

51. Larsen SG, Pfeffer F, Korner H (2019) Norwegian moratorium on transanal total mesorectal excision. Br J Surg 106(9):1120-1121

52. Atallah S, Sylla P, Wexner S (2019) Norway versus The Netherlands: will taTME stand the test of time?. Springer, New York

Publisher's Note Springer Nature remains neutral with regard to jurisdictional claims in published maps and institutional affiliations. 